\title{
Influence of emotional intelligence as the mediator between physical activity and mental health (distress) among Malaysian university students
}

\begin{abstract}
University students typically enter a dynamic transitional period of new independence from their parents that are characterized by many factors. These factors such as social, financial, and environment can be a burden and puts them at risk of mental health distress. Engaging in physical activity has proved to give benefits to mental health. However, not many university students are active during their years at the university. Few psychosocial factors such as emotional intelligence were seen to increased students physical activity and decrease mental distress. Therefore, the purpose of the study is to see whether emotional intelligence can mediate the relationship between physical activity and mental health (distress) among university students at Universiti Putra Malaysia, Malaysia. A correlation study with path analysis was conducted on 266 students at Universiti Putra Malaysia. Few instruments were used such as International Physical Activity Questionnaire SF (IPAQ-SF), General Health Questionnaire-28 (GHQ-28), and Schutte Self Report Emotional Intelligence Test (SSEIT) was used in this study. Path analysis was used to explore association between these aspects. Physical activity, mental health distress and emotional intelligence were significantly correlated and emotional intelligence showed partial mediation effect towards the relationship between physical activity and mental health distress $(\mathrm{p}<0.00)$. Thus, there was an association between physical activity with mental health distress, and emotional intelligence partially mediated this relationship. We interpret these novel data to suggest that physical activity can boost mental health and these physical activity-associated of emotional intelligence relevantly gives benefits in mental health. Such data have important implications for both health practice and policy especially in higher education institutions.
\end{abstract}

Keyword: Emotional intelligence; Physical activity; Mental health; Undergraduate students 